\title{
Text Coherence in Translation
}

\author{
Yanping Zheng \\ Department of Foreign Languages, North China Institute of Science and Technology \\ P.O.Box 206, Yanjiao, East of Beijing, 101601, China \\ Tel: 86-10-6159-5099 E-mail: pyzhengaas@yahoo.com.cn
}

\begin{abstract}
In the thesis a coherent text is defined as a continuity of senses of the outcome of combining concepts and relations into a network composed of knowledge space centered around main topics. And the author maintains that in order to obtain the coherence of a target language text from a source text during the process of translation, a translator can utilize the following approaches: retention of the continuity of senses of a text; reconstruction of the target text for the purpose of continuity; coherence complement in translation and the extracting and foregrounding of topic sentences in translation.
\end{abstract}

Keywords: Coherence, Text, Continuity, Reconstruction, Coherence complement, Foregrounding

Coherence concerns the ways in which the components of the textual world, i.e., the configuration of concepts and relations which underlie the surface text, are mutually accessible and relevant. (Beaugrande \& Dressler, 2002) A concept is definable as a configuration of knowledge (cognitive content) which can be recovered or activated with more or less unity and consistency in the mind. Relations are the links between concepts which appear together in a textual world: each link would bear a designation of the concept it connects to. Thus coherence will be envisioned as the outcome of combining concepts and relations into a network composed of knowledge space centered around main topics.

And for further discussion, language users are supposed to know something about meaning and sense. If meaning is used to designate the potential of a language expression (or other sign) for representing and conveying knowledge (i.e., virtual meaning), then they can use sense to designate the knowledge that actually is conveyed by expressions occurring in a text. Many expressions have several virtual meanings, but under normal conditions, only one sense in a text. If the intended sense is not at once clear, non-determinacy is present. A lasting non-determinacy could be called ambiguity if it is presumably not intended, or polyvalence if the text producer did in fact intend to convey multiple senses at the same time. Though not yet well explained, the human ability to discover intended senses and preclude or resolve ambiguities is one of the most amazing and complex processes of communication.

A text is also the record of the thinking process of the language user(s) either in written or in spoken form. It is a semantic unit and pragmatic unit consisting of a group of coherent sentences which are also cohesive within and between the sentences generally. And a text "makes sense" because there is a continuity of senses among the knowledge activated by the expressions of the text. A "senseless" or "nonsensical" text is one in which text receivers can discover no such continuity, usually because there is a serious mismatch between the configuration of concepts and relations expressed and the receivers prior knowledge of the world. This continuity of senses can be defined as the foundation of coherence, being the mutual access and relevance within a configuration of concepts and relations. The configuration underlying a text is the textual world, which may or may not agree with the established version of the "real world", i.e., that version of the human situation considered valid by a society or social group. Note, however, that the textual world contains more than the sense of the expressions in the surface text: cognitive processes contribute a certain amount of commonsense knowledge derived from the participants' expectations and experience regarding the organization of events and situations. Hence, even though the senses of expressions are the most obvious and accessible contribution to the meaningful-ness of texts, they cannot be the whole picture.

It can be safely concluded, therefore, that a coherent text is a continuity of senses of the outcome of combining concepts and relations into a network composed of knowledge space centered around main topics. And this conclusion can be directed towards the process of translation to yield a truthful target text with smoothness.

In order to obtain the coherence of a target language text from a source text during the process of translation, four solutions have been presented in this thesis: retention of the continuity of senses of a text; reconstruction of the target text for the purpose of continuity; coherence complement in translation and the extracting and foregrounding of topic sentences in translation. Such points will be illustrated one by one in the e following.

\section{Retention of the Continuity of Senses of a Text in Translation}

Retention of the continuity of senses of a text refers to leave the continuity of senses of a source text unchanged and on 
the basis of this a translator tries to comprehend and to produce a faithful target text in the process of translation.

Example 1: All this good cheer was plainly too much for human nature, which seems to crave a regular dose of impending doom. That was not missing in 1988, when fears about the environment loomed larger than ever before. Ocean pollution seemed to touch every continent; medical wastes washed up on America's Atlantic beaches, and dying seals on the shores of the North Sea...

The italicised part of the text has been translated as follows:

Zai1988nian ren men dui huanjin de danyou bi yiwang renhe shihou dou gengweijiaju de yinian li, nazhong lingren huanxinguwu de shi ye meiyou quedaio.

While in the following part of the text, the readers can't see any "good cheer"; instead, they can find a lot of problems. Hence, the target text would not be coherent. According to the continuity of senses of the text, a translator can infer from the whole text that the word "that" in the italicised part refers to something bad instead of any "good cheer". After this inferring a coherent target text can be attained as follows:

Chinese version: Dui ren de benxing er yan, hao xinxi shizai shi duo de guofenle, yinwei renmen sihu zongshi xiang tingdao xie mori jijiang lailin de huai xixiao. Zai renmen dui huanjing wenti bi yiwang renhe shihou dou gengjia youxinchongchong de 1988 nian li, dao ye bu fa zheyang de huai xiaoxi. Haiyang wuran sihu yi yangji gege dalu,feiqi de yiyao laji bei chong dao meiguo daxiyang yan an, yanyanyixi de haibao ye piaofu dao beihao de haitanshang. (Li, 2001:163)

Example 2: Mifeng zhe wujian, zui ai laodong. Guangdong tianqi hao, hua you duo, mifeng yi nian sijie dou bu xian zhe. Liang de mi duo, ziji chi de ke youxian. Mei hui ge mi, ta gei ziji liu yi diandian tang, gou tamen chi jiu xing le. Tamen conglai bu zheng, ye bu jijiao shenme, haishi jixu laodong, jixu nianmi, zhengri zhengyue bucilaoku...

English version 1: The bees are industrious while our province has good weather and plenty of flowers. They work the whole year round, and eat only a fraction of the honey they produce. Each time we extract it we leave them a little sugar. They never argue or complain, just go on producing honey day after day. (Volume of Modern Chinese Prose with English Translation, p281)

English version 2: The bees are industrious. They work the whole year round, since our province has warm weather and plenty of flowers. Though they produce much honey, they eat only a fraction of it. Each time we extract it we leave them a little sugar. They never argue or complain, just go on producing honey day after day. (Li, 2001: 166)

By comparison, we can see that the second version is much more faithful to the original text for it retains the continuity of senses and the coherent relationship of the original text.

\section{Reconstruction of the Target Text for the Purpose of Continuity}

Some linguists hold that the Chinese texts are developed in an inductive way while the English ones are developed in a deductive way. They contribute this difference to the thought patterns of the two peoples. The English thought pattern is straightforward while the Chinese thought pattern is spiral.

So a text in English is usually centered around a topic in the form of topic sentence or topic paragraph and then it is developed directly by many sentences in a sequence. To secure coherence the producer usually deduces the topic in such ways as:

By classification development;

By chronological development;

By spatial development;

By definition development;

By exemplification development;

By comparison development;

By contrast development;

By cause-effect development and so on (Xiao, 2002:120).

While a text in Chinese generally adopts the four steps in composition — starting with an introduction, then elucidation of the theme, transition to another view point, and finally summing up (Zhang and Zhang, 1998: 29).

Therefore, there exists certain striking difference in the composition of texts in the two languages.

And translation is not only the process of the interchange of the linguistic symbols between two languages, more often than not, it is the interchange of thinking processes between language users on the basis of the general models in the two languages, especially, it is so when translation is conducted on text level. Consequently, it is quite necessary for a 
translator to conduct certain reconstruction in the process of translation.

Example 3: Pashanhu zongshi yexinbobo de qitu zhanling mei yi cun qiang. Zai na wushu zhi juanqu xiangshang de tengman zhong, you yi zhi jihu panshang le na jianjian de wuding. Zheshi you yizhen feng gualai, ba ta xuan zai le bankongzhong.

Liu Chuan cong chuangkou wang qu, kandao le duimian qiang shang de zhege jingtou, xia yishi de xiao le qilai(1).

"Ni shi teng, wo shi qiang." You yi tian ta dui Xiao Mei shuo(2).

Ta yanzhong de na zhi tengman hua cheng le ta(nv) de xingxiang(3). Zai ta xiang ta(nv) shuo le zhe ju hua zhihou, ta(female) jue qi le zuba zhuan shen zou le(4).

"Wo meiyou biyao mofang dianying zhong de nan zhujue, jiakuai le jiaobu qu zhui ta(female)." Ta xiang(5). Ta liang ye xiang suoyou nianqing de qinglv na yang tiansheng hao duqi, ke mole, zongshi ta(female) lai zhao ta, yiban bu chu yige xingqi. Ta hen you xingxin. Zheci youxie fangchang. Ta na fu ming wei Qiangzhe de youhua hua qu le zhengzheng yige yue de shijian, ke ta(female) hai meiyou lai, lian xing ye meiyou yi feng. (selected from Wall by Zhou Weibo)(6)

English version: Creepers are always ambitiously trying to crawl over every inch of the wall. One of the numerous curling vines had almost crept onto the steep roof of the house when a sudden gust of wind blew it off in mid air.

Liu Chuan happened to catch sight of the scene on the opposite wall through the window, and he couldn't help smiling. In his eyes the vine was transformed into the image of Xiao Mei.

"You are a creeper while I am a wall," he had once said to her. At his remark, she pursed her lips and went away.

"I won't imitate the hero in the film and run after her," he thought.

They used to quarrel quite often just as other young lovers did. But in the end it was always she who would first come to him for reconciliation within a week or so. He had been quite confident that this argument would end similarly. Yet there was something different about this time. During the whole month he spent on his oil painting entitled "The Fitter," she hadn't turned up once nor even sent word. (Song, 2003: 451)

From the source text, it is noted that there is a causal relationship between sentence (3) and sentence (1), so they are put together in the target text. There is a temporal and a weak causal relationship as well between sentence (2) and sentence (4), hence they are translated as one paragraph. The reflection of his is contained in sentence (5), thus translated as an individual paragraph. The rest of the text is a comparison between the past and the present, which has been dealt with as one paragraph in the target text. After the reconstruction of the message in the source text, a logic target text with clear organization has come into existence. From the example we can undoubtedly see the necessity of the reconstruction of the target text for the purpose of continuity.

\section{Coherence Complement in Translation}

The utilization of texts almost certainly involves steady interactions and compromises between the actual text materials being presented, and the participants' prior disposition, according to conditions which, though flexible and variable, are by no means unsystematic. (Beaugrande and Dressler: 2002:136)

What's more, coherence, together with cohesion, is text-centred notion, designating operations directed at the text materials. In addition, it is necessary to require user-centred notions which are brought to bear on the activity of textual communication at large, both by producers and by receivers. And in some cases there exists implicature- the question of how a language user comes to understand more than is actually said - in a text.

Therefore, a translator assumes the responsibility of maintaining the coherence of a target text from the perspective of the receivers for the convenience of the receivers retrieving information from the target text during the process of translation. For the purpose of this, it is necessary for a translator to complement certain elements in the target text for the sake of the receivers.

Example 4: Wo dangshi shi jieguan zhongyang meishu xueyuan de jun daibiao. TingshuoBai Shi laoren shi jiaoshou, meiyue dao xuexiao yici, hua yi zhang hua gei xuesheng kan, zuo shifan biaoyan. You de xuesheng tichu yao ba ta de gongzi ting diao."

Wo shuo: "Zheyang de lao huajia, mei yue lai yici hua yi zhang hua, jiu shi hen da de gong xian. ---"

English version: I was then the military representative at the Central Academy of Fine Arts. I was told that Baishi was a professor there and that he came to the academy once a month. Every time he came he would paint a picture as an example for the students to imitate, but some of them proposed that his salary should be stopped since he came so infrequently.

"For an artist as old as he is, to come once a month and produce a painting is a great contribution," I explained-(Selected Modern Chinese Prose with English Translation.P258) (Quoted from Li, 2001:185) 
The italicized parts in the above example are elements complemented by the translators of the source texts. Such parts have played a key role in creating the coherence of the target text for the reception of the receivers. The target texts would otherwise be difficult for the perception of the readers.

\section{The Extracting and Foregrounding of Topic Sentences in Translation}

The importance of topic sentences in the organization and development of English texts has been covered in section 2 . And it is pointed out that an English text generally would contain at least one topic paragraph or one topic sentence. Such a pattern is familiar to a large number of language users, native and foreign.

And it is advisable for a translator to perceive the importance of the function of topic sentences in translating both English and Chinese texts.

In order to achieve coherence in the target text during the process of translation, topic sentences can be extracted and fore-grounded by the translator from a source text for the purpose of coherence in the target text. Unless topic concepts are activated, the processing of the textual world is not feasible because in many texts there are no control centers to show the main ideas (Beaugrande and Dressler: 2002, 168). And such a treatment in translation will undoubtedly facilitate text receivers' comprehension of the target text.

Example 5: Taiwan gaoyang xie de tongshu xiaoshuo The Complete Story of Hu Хueyan jin ji nian lai zai nei di yi ban zai ban. You xiaoxi shuo, zao ji nian, yi Hu Xueyan shengping shiji wei xiansuo gaibian de dianshiju "Bayue guihua xiang",meiyou biaoxian chu Hu Xueyan linghuo jingshang de jingsui, yi luohou yu shangpin jingji de shidai chaoliu le, yushi xinjin you liangbu zhongduo shangjia rexin touzi de dianshi lianxu ju zhengzai tongshi kaiji paishe. Duici, yiwei mou gognzi de zongjingli zhichu: "Hu Xueyan da xing qi dao, shi zhongguo shang jie de bei ai."

Pingjie yige ren, xian yao liaojie ta shi shenme ren, liaojie ta de lishi. Hu Xueyan, zuji anhui jixi, shengyu 1823 nian, fuqin zao shi, jia ping. Shaonian shi dushu bu duo....

English version: Mr. Hu Xueyan has no doubt become the focus of publishing and show business these years., a bestseller by Gao Yang in Taiwan, has seen many reprints and impressions in China. A TV series based on his life was put on air years ago, which would soon be succeeded by another two series. The reason for the cast of the new series, it is said, is that the previous one, entitled Fragrant Osmanthus In Mid-autumn, failed to present the essence of Hu's business philosophy, and failed to catch up with the tide of commodity economy. So many companies are ardent in sponsoring the series that one general manager in China has pointed out that it is really a tragedy for the Chinese business circle to prettify Hu Xueyan.

It is a tragedy because Hu Xueyan was only a shrewd but not so decent businessman. Born in Jixi, Anhui province in 1823, Hu was brought up in a poor family, with little schooling. Latter he moved to Hangzhou and... (Yao, 2000)

When comparing the target text with the source text, we can see that two topic sentences have appeared at the beginning of each paragraph of the target text. The topic sentences are extracted from each paragraph of the source text and have been foregrounded so that a coherent target text has come into existence. And a logic relation between the two paragraphs has been established by repetition of the word tragedy in the second paragraph of the target text which enhances the continuity of the target text. If the topic sentences were not provided, it would be very hard for a reader to grasp the focus of information of the text.

Example 6: Many man-made substances are replacing certain natural materials because either the quantity of the natural product can not meet our ever-increasing requirements or, more often, because the physical property of the synthetic substance which is the common name for man-made materials, has been chosen and even emphasized so that it would be of the greatest use in the fields in which it is to be applied.

Chinese version: Renzao cailiao tong cheng wei hecheng cailiao. Xuduo renzao cailiao zhengzai daiti mouxie tianran cailiao, zhe huozhe shi youyu tianran chanpin de shuliang buneng manzu riyi zengzhang de xuyao, huozhe wangwang shi renmen xuanze le hecheng cailiao de yixie wuli xingzhi bing jia yi tuchu er zaocheng de. Yinci, hecheng cailiao zai qi yingyong lingyue zhong juyou jida de yongtu.

The first sentence is the topic sentence which is extracted from the source text and foregrouded in the target text. By doing this, the target text becomes a coherent one; the information points of the target text are very clear and easy for the readers to follow.

In a word, a translator, in terms of semantic and cognitive level, can yield a truthful text by the employment of the above-mentioned means: retention of the continuity of senses of a text; reconstruction of the target text for the purpose of continuity; coherence complement in translation and the extract and foregrounding of topic sentences in translation. Such means can serve as the basis of translation on the textual level

\section{References}

Beaugrande R.De \& W. Dressler. ( 2002). Introduction to Text Linguistics. London: Longman. 
Li, Yunxing. (2001). Introduction to Text Translation. Beijing:China Translation and Publishing Corporation.

Song,Tianxi. (2003). A Practical Course on Tranlation between English and Chinese. Beijing:Publishing House of National Defence Industry.

Xiao, Liming. (2002). English-Chinese Comparative Studies \& Translation. Shanghai: Shanghai Foreign Languge Education Press. 2002.

Yao, Jirong. (2000). On the Nature of Text Translation. Chinese Translators Journal, $5^{\text {th }}, 20-22$.

Zhang, Jin \& Zhang Keding. (1998). Information Structure A Comparative Study in English and Chinese. Kaifeng:Henan University Press. 